Proyecciones

Vol. 18, $\mathrm{N}^{\circ}$ 1, pp. 71-76, July 1999

Universidad Católica del Norte

Antofagasta - Chile

\title{
OPTIMALITY OF THE TRACE OF A PRODUCT OF MATRICES
}

\author{
HÉCTOR MIRANDA P. * \\ Universidad de Bío Bí, Concepción-Chile
}

\begin{abstract}
A simple and short proof of the optimality conditions in the John von Neumann trace inequality for singular values is shown. Possible generalizations and special cases are also considered.
\end{abstract}

Key words. trace inequality, singular values.

AMS Subject Classification. 15A45, 15A18

*Research partially supported by FONDECYT 1960754 and Diprode 9830061. 


\section{Introduction.}

Let $A$ and $B$ be $n \times n$ real matrices, then

$$
-\alpha_{1} \beta_{1}-\ldots-\alpha_{n} \beta_{n} \leq \operatorname{tr}\left(A B^{T}\right) \leq \alpha_{1} \beta_{1}+\ldots+\alpha_{n} \beta_{n}
$$

where $\alpha_{1} \geq \ldots \geq \alpha_{n}$ are the singular values of $A$, and $\beta_{1} \geq \ldots \geq \beta_{n}$ the singular values of $B$. Here, $\operatorname{tr}$ denotes trace of a matrix and ${ }^{T}$ its transpose.

This inequality was first proved by John von Neumann [12] and has important applications in the study of certain equations of nonlinear elasticity $[1,4]$, numerical analysis [2, 3] and factor analysis [9].

\section{Main result.}

A short and straighforward proof of the case when the upper and lower bound in (1) are attained will be given. We will find that under this situation, both $A$ and $B$ have simultaneous singular decompositions.

Theorem 1. Let $A, B \in M_{n}$. Then $\operatorname{tr}\left(A B^{T}\right)=\alpha_{1} \beta_{1}+\ldots+\alpha_{n} \beta_{n}$ if and only if there exists $P, Q \in O(n)$ such that $A=P D_{A} Q, B=P D_{B} Q$, where $D_{A}=\operatorname{diag}\left(\alpha_{1}, \ldots, \alpha_{n}\right), D_{B}=\operatorname{diag}\left(\beta_{1}, \ldots, \beta_{n}\right)$, and the singular values are arranged in weakly decreasing order. $O(n)$ denotes the group of $n \times n$ orthogonal matrices.

Proof : The necessity of the conditions for equality is trivial. Suppose now that $\operatorname{tr}\left(A B^{T}\right)=\alpha_{1} \beta_{1}+\ldots+\alpha_{n} \beta_{n}$, and let $R_{i j}(\theta)$ be a rotation matrix, that is, an identity matrix apart from elements $\cos \theta, \sin \theta,-\sin \theta, \cos \theta$ in positions $(i, i),(i, j),(j, i)$ and $(j, j)$, respectively. Then, since $\sup _{R \in O(n)} \operatorname{tr}\left(R A B^{T}\right)=\alpha_{1} \beta_{1}+\ldots+\alpha_{n} \beta_{n}, \operatorname{tr}\left(R_{i j}(\theta) A B^{T}\right)$ achieves a maximum at $\theta=0$.

$$
\operatorname{tr}\left(R_{i j}(\theta) A B^{T}\right)=c_{i i} \cos \theta-c_{i j} \sin \theta+c_{j i} \sin \theta+c_{j j} \cos \theta+\sum_{k \neq i, j} c_{k k}
$$

where $C=A B^{T}=\left(c_{i j}\right)$. Thus, its derivative with respect to $\theta$ vanishes at $\theta=0$, and this implies that $c_{i j}=c_{j i}$. Consequently, $A B^{T}$ is symmetric, and since $\operatorname{tr}\left(A B^{T}\right)=\operatorname{tr}\left(B^{T} A\right), B^{T} A$ is also symmetric. By the singular value decomposition for real matrices, there exist $P_{1}, Q_{1} \in O(n)$ such that 
$P_{1} A Q_{1}=\operatorname{diag}\left(\alpha_{1}, \ldots, \alpha_{n}\right)=D_{A}$. We may assume that the $\alpha_{i}$ are arranged in weakly decreasing order.

$$
\operatorname{tr}\left(A B^{T}\right)=\operatorname{tr}\left(P_{1} A Q_{1} Q_{1}^{T} B^{T} P_{1}^{T}\right)=\operatorname{tr}\left(D_{A} Q_{1}^{T} B^{T} P_{1}^{T}\right)
$$

Thus, without loss of generality we can assume tha $A$ is diagonal. $D_{A} Q_{1}^{T} B^{T} P_{1}^{T}$ and $Q_{1}^{T} B^{T} P_{1}^{T} D_{A}$ are symmetric. We claim that $Q_{1}^{T} B^{T} P_{1}^{T}$ is diagonal. Call $X=\left(x_{i j}\right)=Q_{1}^{T} B^{T} P_{1}^{T}$. Then the symmetry of $D_{A} Q_{1}^{T} B^{T} P_{1}^{T}$ implies that $\alpha_{i} x_{i j}=\alpha_{j} x_{j i}$ and $x_{i j} \alpha_{j}=x_{j i} \alpha_{i}$ is obtained from the symmetry of $Q_{1}^{T} B^{T} P_{1}^{T} D_{A}$. Hence, $\left(\alpha_{i}^{2}-\alpha_{j}^{2}\right) x_{i j}=0$ for all $i, j$. If $\alpha_{i}^{2} \neq \alpha_{j}^{2}$ for $i \neq j$, then $x_{i j}=0$. So, if $D_{A}^{2}$ has distinct diagonal elements, then $X$ must be diagonal. Because the diagonal elements of $X$ are \pm the singular values of $B$ we conclude that $X=Q_{1}^{T} B^{T} P_{1}^{T}=\operatorname{diag}\left( \pm \beta_{\pi(1)}, \ldots, \pm \beta_{\pi(n)}\right)$ with $\pi$ a permutation of $1, \ldots, n$. In the case where $D_{A}^{2}$ has nondistinct diagonal elements $X=Q_{1}^{T} B^{T} P_{1}^{T}$ splits as a direct sum of blocks $X=\operatorname{diag}\left(X_{1}, \ldots, X_{k}\right)$ corresponding to $D_{A}=\operatorname{diag}\left(\alpha_{1} I_{1}, \ldots, \alpha_{k} I_{k}\right)$ with $\alpha_{1}>\alpha_{2}>\ldots>\alpha_{k-1}>\alpha_{k} \geq 0$. Here, each $I_{i}$ is an identity matrix. A simultaneous block diagonal similarity of $D_{A}$ and $X$, with orthogonal diagonal blocks permits us to take $X_{1}, \ldots, X_{k}$ to be diagonal. Thus, $\operatorname{tr}\left(A B^{T}\right)=\operatorname{tr}\left(D_{A} X\right)=\sum_{1}^{n} \alpha_{i}\left( \pm \beta_{\pi(i)}\right)$, but $\operatorname{tr}\left(A B^{T}\right)=\sum_{1}^{n} \alpha_{i} \beta_{i}$, therefore $A=P_{1} D_{A} Q_{1}$ and $Q_{1}^{T} B^{T} P_{1}^{T}=D_{B}$, that is, $A=P_{1} D_{A} Q_{1}$ and $B=P_{1} D_{B} Q_{1}$

The same proof with minor changes works for the lower bound. We have that a necessary and sufficient condition for equality is also a simultaneous singular value decomposition for $A$ and $B$.

Theorem 2. Let $A, B \in M_{n}$. Then $\operatorname{tr}\left(A B^{T}\right)=-\alpha_{1} \beta_{1}-\ldots-\alpha_{n} \beta_{n}$ if and only if there exists $P, Q \in O(n)$ such that $A=P D_{A} Q, B=P D_{B} Q$ where $D_{A}=\operatorname{diag}\left(\alpha_{1}, \ldots, \alpha_{n}\right), D_{B}=\operatorname{diag}\left(\beta_{1}, \ldots, \beta_{n}\right)$, and the singular values are arranged in weakly decreasing order. $O(n)$ denotes the group of $n \times n$ orthogonal matrices.

\section{Comments.}

3.1. If we replace $\operatorname{tr}\left(A B^{T}\right)$ by $\operatorname{tr}(A B)$ in the theorem we do not have a simultaneous singular decomposition for $A$ and $B$, but we have from the proof of the theorem that $A B$ and $B A$ are symmetric. 
3.2. Fan [6] extended the trace inequality to more than two matrices. The trace of a finite product of matrices is less than or equal to the sum of the products of the corresponding singular values of the factors when these are arranged in weakly decreasing order. It is not possible to extend the optimal result shown here to more than two matrices. As an example, consider the matrices

$$
A=\left(\begin{array}{cc}
-1 & 0 \\
0 & 0
\end{array}\right), B=\left(\begin{array}{ll}
0 & 1 \\
1 & 0
\end{array}\right), C=\left(\begin{array}{cc}
0 & 1 \\
-1 & 0
\end{array}\right) .
$$

Then $\operatorname{tr}(A B C)=1$, and the sum of the products of the singular values is also 1 , but $A B$ and $A C$ are not symmetric.

3.3. The optimality of (1) was studied by Kiers and Ten Berge [8] and their proof was strongly dependent on a result by Friedland [7]. According to de Sá [5] this optimality is hidden in the original von Neumann's proof of (1), but this is not that simple. Lewis [10] studied (1) under Lie theoretical terms.

3.4. In the case where the matrices are symmetric positive semidefinite, the singular values in (1) are replaced by eigenvalues. Theobald [11] used the structure of doubly stochastic matrices to give a proof of the optimality in this case. Our proof also works to produce:

Corollary 1. If $A$ and $B$ are symmetric positive semidefinite matrices the inequality (1) becomes

$$
\operatorname{tr}(A B) \leq \alpha_{1} \beta_{1}+\ldots+\alpha_{n} \beta_{n}
$$

where the alpha and beta are now the eigenvalues of $A$ and $B$ respectively. In this case the upper bound is attained if and only if there exists $P \in$ $O(n)$ such that $A=P D_{A} P^{T}$ and $B=P D_{B} P^{T}$, that is, $A$ and $B$ have simultaneous spectral decompositions.

3.5. It is not possible to extend the trace inequality in the real symmetric case to more than two matrices as Fan did it in the arbitrary case. For instance, consider the matrices

$$
A=\left(\begin{array}{ll}
3 & 1 \\
1 & 3
\end{array}\right), B=\left(\begin{array}{cc}
2 & -1 \\
-1 & 2
\end{array}\right), C=\left(\begin{array}{cc}
-1 & 0 \\
0 & -1
\end{array}\right) .
$$

Then $\operatorname{tr}(A B C)=-10$, but the sum of the product of the eigenvalues is -14 . 


\section{References}

[1] J. M. Ball, Convexity conditions and existence theorems in nonlinear elasticity, Arch. Rat. Mech. Anal., 63, pp. 337 - 403, (1977).

[2] R. W. Brockett, Dynamical systems that sort lists, diagonalize matrices, and solve linear programming problems, Linear Algebra Appl., 146, pp. 79-91, (1991).

[3] R. W. Brockett, Differential geometry and the design of gradient algorithms, Proceedings of Symposia in Pure Mathematics, 54 (1993), pp. 69-92.

[4] P. G. Ciarlet, Mathematical Elasticity, Vol. I, North-Holland, Amsterdam, (1988).

[5] E. M. de Sá, Exposed faces and duality for symmetric and unitarily invariant norms, Linear Algebra Appl., 197/198, pp. 429-450, (1994).

[6] K. Fan, Maximum properties and inequalities for the eigenvalues of completely continuous operators, Proc. Nat. Acad. Sci. U.S.A., 37, pp. $760-766,(1951)$.

[7] S. Friedland, Inverse eigenvalue problems, Linear Algebra Appl., 17, pp. $15-51,(1977)$.

[8] H. A. L. Kiers and J. M. F. Ten Berge, Optimality conditions for the trace of certain matrix products, Linear Algebra Appl., 126 (1989), pp. 125-134.

[9] W. Kristof, $A$ theorem on the trace of certain matrix products and some applications, J. Math. Psych., 7, pp. 515 - 530, (1970).

[10] A. S. Lewis, Von Neumann's lemma and a Chevalley-type theorem for convex functions on Cartan subspaces, Research Report, Department of Combinatorics and Optimization, University of Waterloo, (1995).

[11] C. M. Theobald, An inequality for the trace of the product of two symmetric matrices, Math. Proc. Camb. Phil. Soc., 77, pp. 265 - 267, (1975). 
[12] J. von Neumann, Some matrix inequalities and metrization of matrix space, Tomsk. Univ. Rev. 1, pp. $286-300$, (1937). Collected Works, Vol 4, Pergamon, Oxford, pp. $205-219$, (1962).

Received : January 20, 1999.

Héctor Miranda

Departamento de Matemáticas

Universidad de Bío Bío

Casilla 5 - C

Concepción

Chile 